\title{
Simulation of CBRAM devices with the level set method
}

\author{
P.Dorion $^{1,2}$, O.Cueto ${ }^{1}$, M.Reyboz ${ }^{1}$, E.Vianello ${ }^{1}$, J.C.Barbé ${ }^{1}$, A.Grigoriu ${ }^{3}$, Y.Maday ${ }^{2}$ \\ ${ }^{1}$ CEA-LETI MINATEC, 17 rue des Martyrs, 38054 Grenoble, Cedex 9, France \\ ${ }^{2}$ Laboratoire J.-L Lions, Université Pierre et Marie Curie, F-75005, Paris, France \\ ${ }^{3}$ Laboratoire J.-L Lions, Université Paris Diderot, F-75013, Paris, France \\ pierre.dorion@cea.fr, Phone: +3343878 2340
}

\begin{abstract}
A TCAD model for Chalcogenide based CBRAM is introduced. This model relies on the level set method to follow the growth of the filament in the electrolyte. We couple the level set method with physical equations which model the cations migration and the electric field in the electrolyte and in the filament. The formation and the dissolution of the filament can be simulated. Comparisons between simulations and electrical results are used to validate our model.
\end{abstract}

\section{INTRODUCTION}

Conducting-Bridge Random-Access Memory (CBRAM) is a promising technology for future nonvolatile memories, due to its low operating voltages, low power consumption and ease of integration in the back end of a logic process. A CBRAM cell is composed of a resistive switching layer encapsulated between an electrochemically active electrode, and an electrochemically inert counter electrode. The storage of the information is based on the contrast between a high resistance state and a low resistance state. Resistance switching is induced by electro-chemical driven growth and rupture of a metallic filament in the electrolyte. A new simulation tool based on the finite element discretization of a system of partial differential equations is presented in this work. During write operation (SET), cations obtained from the oxidation of the top electrode migrate through the electrolyte, reduce on the filament and contribute to filament growth until it connects the two electrodes. The erase operation (RESET) is achieved by the electro-chemical driven dissolution of the filament. The Level Set Method (LSM) is used to simulate the filament growth and dissolution.

\section{PHYSICAL MODEL}

\section{A. SET mechanisms}

Our CBRAM model relies on assumptions widely used [1] - [2]. We study a CBRAM cell based on an active $A g$ top electrode, a $G e S_{2}$ electrolyte and a $W$ inert electrode illustrated by Fig. 1. In all samples, a thin $A g$ layer has been dissolved into the $\mathrm{GeS}_{2}$ electrolyte using a photo-diffusion process. Our model would be easily extended to other types of CBRAM based on cation migration.

Under a sufficient positive voltage on the active electrode, the following mechanisms leading to the SET occur:

(i) Anodic dissolution by Ag oxidation:

$$
A g \longrightarrow A g^{+}+e^{-}
$$

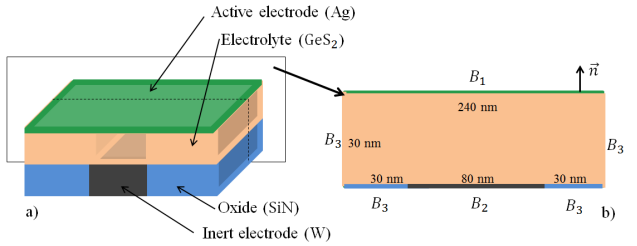

Fig. 1: a) 3D representation of study device, b) domain of resolution with the dimensions of our cell and the definition of the boundaries. The vector $\vec{n}$ defines the normal at the boundary used in the expression of the boundaries conditions.

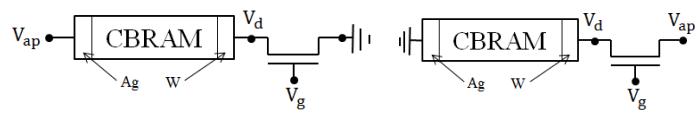

(a)

(b)

Fig. 2: Schematics of CBRAM model 2a. For the SET operation including a transistor coupled with the CBRAM device via $V_{d}$ floating potential node, $2 \mathrm{~b}$. For the RESET operation.

(ii) Migration of the $\mathrm{Ag}^{+}$cations through the solid electrolyte under the action of the electric field and the gradient of concentration.

(iii) Reduction and electrocrystallization of $\mathrm{Ag}$ on the surface of the inert electrode and the filament according to the reaction: $\mathrm{Ag}^{+}+e^{-} \longrightarrow A g$.

Oxidation reaction is modeled with a Butler-Volmer equation which describes the current density across the electrode interface as a function of an exchange current density $j_{0}$, a charge transfert coefficient $\alpha$ and an overvoltage $\eta$ :

$$
J_{B V}=j_{0}\left(\exp \left[\frac{\alpha e \eta}{k_{B} T}\right]-\exp \left[\frac{-(1-\alpha) e \eta}{k_{B} T}\right]\right)
$$

where, $k_{B}$ is the Boltzmann constant, $T$ is the temperature and $e$ is the elementary charge. For the SET, the overvoltage $\eta$ is the difference between the applied voltage $V_{a p}$ and the Nernst potential $\eta_{0}$. For the RESET model, we use the equation (1) to model the oxidation on the filament. So we express the overvoltage by $\eta=V_{d}-\eta_{0}$ where $V_{d}$ is the voltage on the inert electrode illustrated by Fig. $2 \mathrm{a}$. 
We assume the Ag electrode is an infinite silver reservoir. So, only the evolution of the Ag based conductive filament in the electrolyte is modeled. We assume that the nucleation is not the limiting step in the formation of the filament. So our SET model assumes the existence of a critical nucleus on the inert electrode from which the filament starts growing. The typical thickness of electrolyte-insulator films in our CBRAM devices varies between 10 and $100 \mathrm{~nm}$ which is often comparable or even smaller than charge screening length. This results in a situation where the local charge neutrality fails. So we do not make the assumption of bulk electroneutrality widely used in the simulation of electrochemical systems and work instead with the Poisson-Nernst-Planck equations allowing for non constant concentration of charges in solution. We consider space charge in our solid electrolyte is reduced to $\mathrm{Ag}^{+}$cations and we calculate ionic concentration and electrostatic potential with the equations:

$$
\begin{aligned}
& e \frac{\partial C_{A g^{+}}}{\partial t}-\operatorname{div}\left(e D \nabla C_{A g^{+}}+\sigma \nabla V\right)=f \mathbf{1}_{b d} \\
& \left\{\begin{aligned}
\left(e D \nabla C_{A g^{+}}+\sigma \nabla V\right) \cdot \vec{n} & =J_{B V} & & \text { on } B_{1} \\
\left(e D \nabla C_{A g^{+}}+\sigma \nabla V\right) \cdot \vec{n} & =-v_{g} e C_{A g^{+}} & & \text {on } B_{2} \\
\left(e D \nabla C_{A g^{+}}+\sigma \nabla V\right) \cdot \vec{n} & =0 & & \text { on } B_{3}
\end{aligned}\right. \\
& -\operatorname{div}(\epsilon \nabla V)=e C_{A g^{+}} \\
& \left\{\begin{array}{clrl}
V & =V_{a p} & & \text { on } B_{1} \\
V & =V_{d} & & \text { on } B_{2} \\
\nabla V \cdot \vec{n} & =0 & & \text { on } B_{3}
\end{array}\right.
\end{aligned}
$$

The equation (2) models the charge conservation and the Poisson equation (4) couples the electric field and the local charge density. The equation (3) and (5) define the boundaries conditions of our devices, the boundaries are illustrated by Fig. 1. The parameters $V, C_{A g^{+}}, \epsilon, \sigma$ and $D$ represent respectively the electric potential, the concentration of $\mathrm{Ag}^{+}$, the permittivity, the conductivity and the coefficient of diffusion of $\mathrm{Ag}^{+}$. The term $f$ in equation (2) models the absorption on the surface of the filament and $\mathbf{1}_{b d}$ is an interface filamentelectrolyte indicator function. In the SET model, we take $f=10^{30} e C_{A g^{+}}$to modelize the reduction of ion $\mathrm{Ag}^{+}$at the surface of the filament. We know that part of dissolved $\mathrm{Ag}$ atoms are ionized, so our cells contain initially $\mathrm{Ag}^{+}$ cations which define the initial value for $C_{A g^{+}}$. The velocity of filament growth $v_{g}$ is presented from the down of the document. We model the ionic conductivity with a non-linear expression of an average electric-field $E_{m}=V_{a p} / H_{c e l l}$ in the electrolyte

$$
\sigma=\frac{s_{0}}{E_{m}} \sinh \left(\frac{a\left(E_{m}\right) e E_{m}}{2 k_{B} T}\right)
$$

where $s_{0}$ is a prefactor including temperature dependency considered constant for our study, $H_{c e l l}$ is the electrolyte height and $a$ is the jump distance of the ions. Our model corresponds to a drift density of current $\vec{j}_{\mathrm{Ag}^{+}}=\sigma E$ inspired by the model of Mott and Gurney [3] for an electric field driven ion hopping. We must introduce a dependency of $a$ on the average electric field to calibrate our conductivity model. Dependency of $a$ on the electric field shows no real physical meaning which renders the possibility of non-linear ion migration less likely [1].

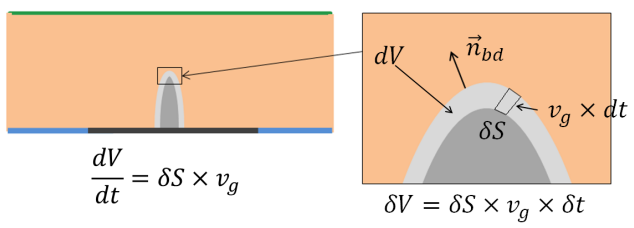

Fig. 3: Schematics of the variation of volume $d V$ during deposition step. A spatial discretisation of this variation $\delta V$ is expressed by considering a small surface $\delta S$ moved during a small time $\delta t$ at the velocity $v_{g}$.

\section{B. RESET mechanisms}

The RESET operation includes the physical mechanisms which erase the filament between the two electrode. It begins by applying a negative voltage on the top electrode or a positive voltage on the inert electrode. The oxydation of $\mathrm{Ag}$ atoms occurs on the surface of the filament. The migration of the cations $\mathrm{Ag}^{+}$is still under the electric field effects and the reduction of the cations occurs on the silver electrode. Our physical modelisation of the RESET uses the same equations than the SET model. The difference is in the expression of $f$ which modelizes the oxydation of the filament with a ButlerVolmer equation (1).

\section{Electrocrystallization and growth of the filament}

The electrocrystallization involves the initial nucleation of $\mathrm{Ag}$ in the inert electrode and the subsequent filament growth. The relation between the deposited volume and the deposition current follows the Faraday law:

$$
\frac{d \mathcal{V}}{d t}=I(t) \frac{\mathcal{V}_{A g}}{e}
$$

where $\mathcal{V}_{A g}=M_{A g} / \rho_{A g}$ is the volume of an Ag atom, $M_{A g}$ is the atomic mass and $\rho_{A g}$ is the density of Ag. The term $I(t)$ represents the charge current though the interface. We consider the volume change, illustrated by Fig. 3, can be discretized in space by a velocity field $\vec{v}$ which moves a little surface of the interface during a small time $\delta t$ according to the normal of the interface $\vec{n}_{b d}$ :

$$
\frac{\delta \mathcal{V}}{\delta t} \approx \delta S \vec{v} \cdot \vec{n}_{b d}
$$

where $\delta S$ represents the surface moved by the velocity field $\vec{v}$ according to the normal at the interface $\vec{n}_{b d}$. The scalar product $v_{g}=\vec{v} \cdot \vec{n}_{b d}$ represents the velocity of filament growth which is used in equation (3). The parameter $\delta t$ is the time step. So, by using (7) and (8) we obtain

$$
v_{g}=\frac{I(t)}{\delta S} \frac{\mathcal{V}_{A g}}{e}
$$

The term $I(t) / \delta S$ corresponds to the flux of charge close to the surface $\delta S$.

We consider that nucleation time is not the rate limiting step. The switching kinetics may be controlled by diffusion or by charge transfer kinetics at interface. Our growth filament model is able to take into account both limitations. In the first case, the flux of charges is expressed by

$$
\frac{I(t)}{\delta S}=\vec{j}_{A g^{+}} \cdot \vec{n}_{i n t} \Rightarrow v_{g}=\frac{\mathcal{V}_{A g}}{e} \vec{j}_{A g^{+}} \cdot \vec{n}_{i n t}
$$




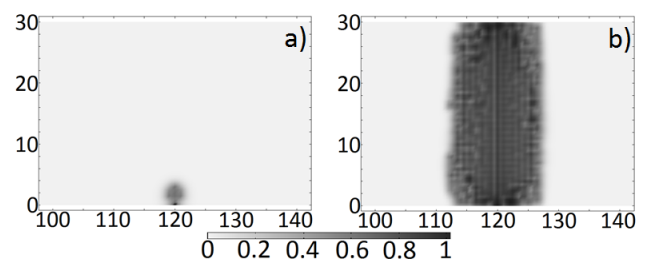

Fig. 4: Results of SET model simulation with the stabilization term in (13). a) Filament at time $\mathrm{t}=2 \mu \mathrm{s}$, b) filament at the end of the SET $(9 \mu \mathrm{s})$.

This expression of the velocity implies that the ions $\mathrm{Ag}^{+}$ which are close to the filament catch instantaneously an electron, so electron transfer is faster than mass transfer. In the second case, the flux of charges is expressed by the ButlerVolmer equation (1)

$$
\frac{I(t)}{\delta S}=j_{B V} \Rightarrow v_{g}=j_{B V} \frac{\mathcal{V}_{A g}}{e}
$$

Inside the electrolyte, the filament area corresponds to a domain with higher electrical conductivity; physical coefficients of our model have point value depending on the position in the electrolyte.

\section{LEVEL SET METHOD}

\section{A. Definition}

LSM introduced by Osher and Sethian [4] is a versatile method used in many problems with an interface motion [5]. It allows including topological changes without explicit front tracking method and thus provides considerable gain in both algorithmic complexity and CPU time. This method implies a space-time function $\varphi$ with output between 0 and 1 . The interface between filament and electrolyte is defined by the level set $\{\varphi=0.5\}$. The level set function $\varphi$ is solution of an advection equation:

$$
\frac{\partial \varphi}{\partial t}+\vec{v} \cdot \nabla \varphi=0
$$

where $\vec{v}$ represents the velocity of filament growth.

\section{B. Stabilisation of advection-equation}

The advection equation classically needs a stabilization term to prevent instabilities in the numerical simulation. We used a stabilization method which was applied by Olsson [6] in two-phase flow simulation. The stabilized advection equation can be expressed as

$$
\frac{\partial \varphi}{\partial t}+\vec{v} \cdot \nabla \varphi=\gamma \operatorname{div}\left(\xi \nabla \varphi-\varphi(1-\varphi) \frac{\nabla \varphi}{\|\nabla \varphi\|}\right)
$$

where $\gamma=v_{g}$ and $\xi=h$ the characteristic mesh size.

The stabilization term used at the right member of the advection equation (13) allows simulating both the electrical field in the entire device and the filament growth (cf Fig. 4).

Another choice for $\gamma$ and $\xi$ give more instabilities in numerical results illustrated by Fig. 5. These instabilities increase the CPU time, so we choose $\gamma$ and $\xi$ via a tradeoff between CPU time and stability.

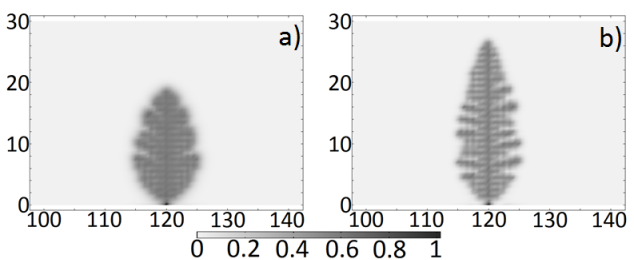

Fig. 5: Results of SET model with differents values for $\xi$ and $\gamma$ at time $t=7 \mu \mathrm{s}$ with the same applied voltage a) $\xi=h$ and $\gamma=v_{g}$ which are the chosen values, b) $\xi=h / 2$ and $\gamma=2 v_{g}$.

TABLE I: Parameters used in the simulation.

\begin{tabular}{|c|c|c|c|}
\hline Parameter & Value & Parameter & Value \\
\hline$\eta_{0}$ & $0.23 \mathrm{~V}$ & $j_{0}$ & $10^{-2} \mathrm{~A} \cdot \mathrm{m}^{-2}$ \\
\hline$D$ & $1.710^{-7} \mathrm{~m}^{2} \cdot \mathrm{s}^{-1}$ & $D_{0}$ & $10^{-2} \mathrm{~m}^{2} \cdot \mathrm{s}^{-1}$ \\
\hline$s_{0}$ & $10^{5} \mathrm{~A} \cdot \mathrm{m}^{-2}$ & $H_{\text {cell }}$ & $30 \mathrm{~nm}$ \\
\hline$\rho_{A g}$ & $10490 \mathrm{~kg} \cdot \mathrm{m}^{-3}$ & $\epsilon_{\text {elec }}$ & $10^{6} \epsilon_{0}$ \\
\hline$E_{a}$ & $1.6 \mathrm{eV}$ & $\epsilon_{f i l}$ & $7 \epsilon_{0}$ \\
\hline$M_{A g}$ & $1.7910^{-21} \mathrm{~g} . a t^{-1}$ & $\alpha$ & 0.158 \\
\hline
\end{tabular}

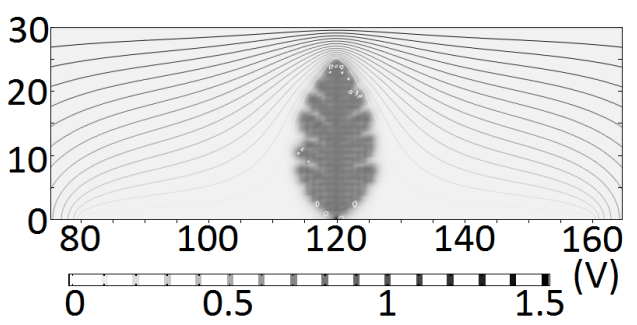

Fig. 6: Electric Potential at time $\mathrm{t}=8 \mu \mathrm{s}$ obtained with the Poisson equation (4). The isovalues of the potential show the electrical neutrality of the filament.

\section{RESULTS}

\section{A. Implementation}

Our model is implemented using software Comsol. Equations (2), (4) and (13) are discretized using quadratic Lagrange finite element on a cartesian mesh with a characteristic size of $0.5 \mathrm{~nm}$. The constants used in simulations are given in Tab.1. We simulate the system in a $2 \mathrm{D}$ domain corresponding to perpendicular cut plane of the inert electrode in a 3D structure (cf Fig. 1). The relative permittivity in the filament is chosen large enough to model electrical neutrality inside the filament. In the equation (13), the level set velocity $\vec{v}$ is expressed by the relation $\vec{v}=v_{g} \vec{n}_{b d}$ where $v_{g}$ is the velocity of filament growth given in equation (10). We verify electrical neutrality inside the filament as shown in Fig. 6.

\section{B. Comparison with electrical results and discussion}

We present comparisons with electrical results obtained on $G e S_{2}$ CBRAM devices [7]. We couple our system with a transistor current model to introduce compliance current (cf Fig. 2a) during the SET. The compliance current allows to stop the growth of the filament by limiting the current in the device. If we don't use it, the filament keeps growing and we can not erase it during the RESET. For the RESET, we apply an electrical potential on the inert electrode. 


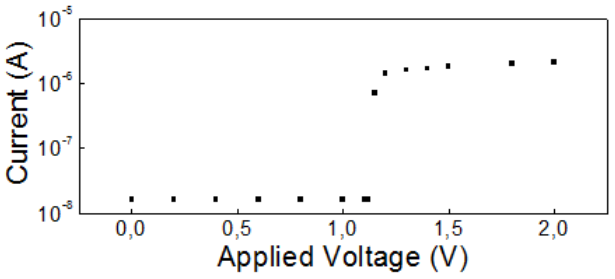

Fig. 7: I-V curve obtained numerically. We observe a saturation of the current under high applied voltage thanks to the coupling of the equations (2)-(5) with a current transistor model.

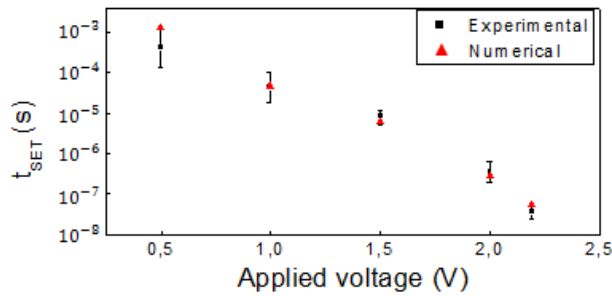

Fig. 8: Experimental and numerical switching time $t_{S E T}$ for devices with a chalcogenide $G e S_{2}$ electrolyte doped $10 \% \mathrm{Sb}$. The model of ionic conductivity (6) allows having a good approximation of the switching time.

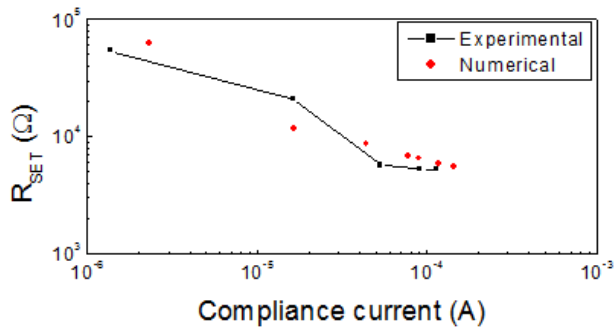

Fig. 9: Resistance after SET $R_{S E T}$ versus saturated current delivered by the transistor for $\mathrm{GeS}_{2} 10 \%$ Sb devices.

First, we apply different constant voltage pulses during the same time to obtain a representation of the current in the device as a function of the applied voltage (cf Fig. 7).

Our model for the cations conductivity as a function of electric field (6) allows having a good approximation of the switching time $t_{S E T}$ (cf Fig. 8) as a function of applied voltage.

We fix the value of the filament electrical conductivity by approaching the data on SET resistance versus compliance current (cf Fig. 9). The decrease of the resistance while increasing the compliance current is explained with our model if we assume a gradual increase in the radius of the conductive filament formed during the set-process.

Finally, we simulate temperature-accelerated retention experiments. We believe that the retention loss mechanism for the $\mathrm{ON}$ state is due to the diffusion of the $\mathrm{Ag}$ atoms forming the conductive filament is the surrounding solid electrolyte [7]. We model the retention with a diffusion equation and fix the value of the thermal-activated $\mathrm{Ag}$ diffusion in the electrolyte

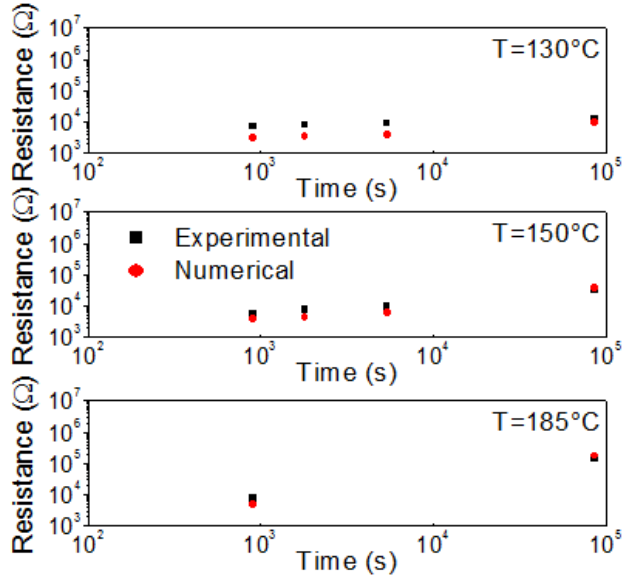

Fig. 10: Retention Characteristics at high temperature for $\mathrm{GeS}_{2}$ $10 \%$ Sb CBRAM.

(cf Fig. 10) by fitting experimental retention curves:

$$
D_{t h}=D_{0} \exp \left(-\frac{E_{a}}{k_{B} T}\right)
$$

\section{CONCLUSION AND PERSPECTIVES}

The formation and the dissolution of the filament in a CBRAM device can be simulated with our model. 2D simulation results reproduce well the experimental results. Filament growth will be further studied by taking into account charges transfer limitation. Extension to 3D simulation would be natural because the level set method extends naturally to 3D. A stochastic character will be added to our model by taking into account nucleation. The model will be extended with the Fourier equation, in order to study thermal effects in CBRAM devices. An alternative to the contact ohmic model between the filament and the active electrode will be introduced through a tunneling current model.

\section{ACKNOWLEDGMENT}

The authors would like to thank P. Blaise for fruitful discussions and A. Glière for sharing Comsol experience.

\section{REFERENCES}

[1] R.Waser, R.Dittmann, G.Staikov, and K.Szot. Redox-based resistive switching memories - nanoionic mechanisms, prospects, and challenges, partie 1-3. Advanced Materials, pages 2632-2663, 212009.

[2] S.Menzel, B.Klopstra, C.Kgeler, U.Bttger, G.Staikov, and R.Waser. A simulation model of resistive switching in electrochemicalmetallization memory cells. Materials Research Society, 1160:101-106, 2009.

[3] N.F.Mott and R.W.Gurney. Electronic Processes in Ionic Crystals, Second Edition. Oxford, 1948.

[4] S.Osher and J.A.Sethian. Fronts propagating with curvature dependent speed: Algorithms based on hamilton-jacobi formulations. Journal of Computational Physics, 79:12-49, 1988.

[5] M.F. Gyure et al. Level set methods for the simulation of epitaxial phenomena. Physical Review E, 58(6):6927-6930, 1998.

[6] E.Olsson, G.Kreiss, and S.Zahedi. A conservative level set method for two phase flow II. Journal of Computational Physics, 225:785-807, 2007.

[7] E. Vianello et al. Sb-doped $G e S_{2}$ as performance and reliability booster in Conductive Bridge RAM. IEDM, 2012. 